\title{
Microwave analysis of the interplay between magnetism and superconductivity in $\operatorname{EuFe}_{2}\left(\operatorname{As}_{1-x} P_{x}\right)_{2}$ single crystals
}

\author{
G. Ghigo $₫,{ }^{1,2, *}$ D. Torsello $\odot,{ }^{1,2}$ L. Gozzelino, ${ }^{1,2}$ T. Tamegai, ${ }^{3}$ I. S. Veshchunov, ${ }^{4,3}$ S. Pyon, ${ }^{3}$ W. Jiao, ${ }^{5}$ G.-H. Cao ${ }^{5},{ }^{5}$ \\ S. Yu. Grebenchuk, ${ }^{4}$ I. A. Golovchanskiy ${ }^{\circ},{ }^{4}$ V. S. Stolyarov ${ }^{\circ},{ }^{4}$ and D. Roditchev ${ }^{6}$ \\ ${ }^{1}$ Department of Applied Science and Technology, Politecnico di Torino, Torino 10129, Italy \\ ${ }^{2}$ Istituto Nazionale di Fisica Nucleare, Sezione di Torino, Torino 10125, Italy \\ ${ }^{3}$ Department of Applied Physics, The University of Tokyo, Hongo, Bunkyo-ku, Tokyo 113-8656, Japan \\ ${ }^{4}$ Moscow Institute of Physics and Technology (State University), Dolgoprudny, Moscow 141700, Russia \\ ${ }^{5}$ Department of Physics, Zhejiang University, Hangzhou 310027, China \\ ${ }^{6}$ Laboratoire de Physique et d'Etude des Materiaux, UMR8213, \\ École supérieure de physique et de chimie industrielles de la Ville de Paris, Paris Sciences et Lettres Research University, \\ Institut des NanoSciences de Paris-Sorbonne Universite, 10 rue Vauquelin, Paris 75005, France
}

(Received 5 July 2019; published 18 November 2019)

\begin{abstract}
We report on the microwave analysis of the interplay between magnetism and superconductivity in single crystals of $\mathrm{EuFe}_{2}\left(\mathrm{As}_{1-x} \mathrm{P}_{x}\right)_{2}$, accomplished by means of a coplanar waveguide resonator technique. The bulk complex magnetic susceptibility $\chi_{m}$ extracted through a cavity perturbation approach is demonstrated to be highly sensitive to the magnetic structure and dynamics, revealing two distinct magnetic transitions below the superconducting critical temperature. By a comparison with magnetic force microscopy maps, we ascribe the $\chi_{m}^{\prime \prime}$ peak observed at about $17 \mathrm{~K}$ to the transition from the ferromagnetic domain Meissner phase to the domain vortex-antivortex state, with the subsequent evolution of the domain structure at lower temperatures. The second $\chi_{m}^{\prime \prime}$ peak observed at $11 \mathrm{~K}$ reflects a specific high-frequency feature, connected to vortex-antivortex dynamics and eventual spin reorientation transition of the $\mathrm{Eu}^{2+}$ canted ferromagnetic subsystem. The two peaks merge and vanish upon application of an in-plane magnetic field, which is compatible with the presence of a quantum critical point below $1 \mathrm{~T}$.
\end{abstract}

DOI: 10.1103/PhysRevResearch.1.033110

\section{INTRODUCTION}

The interplay between superconductivity and magnetism is currently one of the most relevant and intriguing topics in condensed matter physics. The interplay is twofold: It is well known that magnetism competes with superconductivity, but in several systems it could be involved in the mechanism of superconductivity itself, as in the case of cuprates, strongly correlated heavy fermions, and iron-based superconductors. In particular, it has been shown that magnetic moments in the FeAs layers of iron-based pnictides have a tendency to order antiferromagnetically and it has been theorized that AFM spin fluctuations can induce the $s_{ \pm}$pairing at the origin of superconductivity in these compounds [1]. This picture, although not completely accepted, has been suggested for several compounds of the so-called 122 family, i.e., $\mathrm{BaFe}_{2} \mathrm{As}_{2}$ with various substitutions, isovalent or inducing electron or hole doping [2-4]. Within this framework, systems also containing

\footnotetext{
${ }^{*}$ Corresponding author: gianluca.ghigo@ polito.it

Published by the American Physical Society under the terms of the Creative Commons Attribution 4.0 International license. Further distribution of this work must maintain attribution to the author(s) and the published article's title, journal citation, and DOI.
}

magnetic rare-earth-metal elements are of interest, since at low temperatures they develop additional magnetic ordering of local moments. EuFe $\mathrm{As}_{2}$-based systems are particularly interesting because of the proximity of superconducting and ferromagnetic transition temperatures, where the latter is connected to the $\mathrm{Eu}^{2+}$ local magnetic moments. In some compounds of this family, the superconducting critical temperature is even higher than that of the magnetic ordering $[5,6]$.

In the $\mathrm{EuFe}_{2} \mathrm{As}_{2}$ parent compound, besides Fe moments aligned along the $a$ direction and ordered antiferromagnetically in both $a$ and $c$ directions, the $\mathrm{Eu}^{2+}$ moments are aligned along the $a$ direction and ordered antiferromagnetically along the $c$ direction [7]. Superconductivity can be induced in this system by chemical pressure through the isovalent substitution of $\mathrm{P}$ in the site of $\mathrm{As}$ [8]. In the $\mathrm{EuFe}_{2}\left(\mathrm{As}_{1-x} \mathrm{P}_{x}\right)_{2}$ compound, it was found that with increasing $\mathrm{P}$ substitution, the magnetic moments of the $\mathrm{Eu}^{2+}$ ions cant out of the $a b$ plane, yielding a net ferromagnetic component along the $c$ direction. Moreover, a possible interplay between $\mathrm{Eu}$ and Fe magnetic sublattices should be considered, since the spin reorientation of $\mathrm{Fe}$ below the $\mathrm{AFM}$ ordering temperature of $\mathrm{Eu}$ was recently found in $\mathrm{Eu}_{0.5} \mathrm{Ca}_{0.5} \mathrm{Fe}_{2} \mathrm{As}_{2}$ [9].

A rich phenomenology of magnetic phases is observed in these systems, where superconductivity coexists with canted ferromagnetism, although a general consensus about their phase diagram has not been established yet. Zapf et al. [5] 
observed in $\mathrm{EuFe}_{2}\left(\mathrm{As}_{1-x} \mathrm{P}_{x}\right)_{2}$ two distinct magnetic transitions below the superconducting transition temperature $T_{s c}$, and concluded that magnetic ordering at a higher temperature is associated with the predominant antiferromagnetic interlayer coupling, whereas the transition at lower temperature is identified as the changeover to a spin glass state, where the moments between the layers are decoupled. Recently, a transition to a nanometer-scale striped domain structure upon cooling below $T_{s c}$ has been reported [10,11]. At yet lower temperature and without any externally applied magnetic field, the system spontaneously undergoes a phase transition into a domain vortex-antivortex state characterized by domains with peculiar patterns. A theory of such transitions was elaborated by Devizorova et al. [12], who calculated the temperature evolution of the magnetic texture, also accounting for the hysteretic behavior revealed by the experiment when varying temperature.

In this work, we report on the microwave response of $\mathrm{EuFe}_{2}\left(\mathrm{As}_{1-x} \mathrm{P}_{x}\right)_{2}$ single crystals and compare it with the local magnetic response as revealed in magnetic force microscopy (MFM) maps. Since time-resolved magneto-optical measurements in the same system revealed a slow relaxation time for $\mathrm{Eu}^{2+}$ spin, with $\tau^{-1} \approx 10 \mathrm{GHz}$ [13], a probe operating in this frequency range seems to be particularly sensitive to spin dynamics, suitable to detect changes in the magnetic structure of the material. Moreover, with respect to dc and low-frequency responses already reported, it enables the exploration of different dynamical regimes. In particular, we used a coplanar waveguide resonator (CPWR) technique, useful to detect the complex magnetic susceptibility and the penetration depth of small and thin crystals [14-17]. We clearly observed, below the superconducting transition, the onset of magnetic ordering followed, at lower temperatures, by two distinct transitions, detected as two clear peaks in the microwave signal. A comparison with MFM maps allowed us to speculate about the origin of these rf features. We associate one of them to the transition from the ferromagnetic domain Meissner phase to the domain vortex-antivortex state. The second one, since there is no direct correspondence with quasistatic MFM and with dc standard magnetic chracterization, can be assigned to a high-frequency mechanism, i.e., a change in the vortexantivortex dynamics, and eventual spin reorientation transition of the $\mathrm{Eu}^{2+}$ canted ferromagnetic subsystem. Finally, since we observed that both the phase transitions can be suppressed by an external in-plane field, we discuss the possible presence of a quantum critical point (QCP).

\section{EXPERIMENTAL DETAILS}

Single crystals of $\mathrm{EuFe}_{2}\left(\mathrm{As}_{1-x} \mathrm{P}_{x}\right)_{2}$ were grown by a selfflux method $[18,19]$. Because of the narrow superconducting dome in the $\mathrm{EuFe}_{2}\left(\mathrm{As}_{1-x} \mathrm{P}_{x}\right)_{2}$ phase diagram [6], crystals with slightly different doping $-x=0.20$ and 0.23 , grown using different precursors (Eu, Fe, P, As and Eu, FeAs, FeP, respectively) - show significantly different critical temperatures, $T_{s c}=24.1 \mathrm{~K}$ and $22.3 \mathrm{~K}$, respectively. For a comparison, we also measured a $\mathrm{BaFe}_{2}\left(\mathrm{As}_{1-x} \mathrm{P}_{x}\right)_{2}$ crystal with $x=0.33$, showing $T_{s c} \approx 29 \mathrm{~K}$. The investigated samples were cleaved and shaped in the form of thin plates with thickness of the order of $10 \mu \mathrm{m}$, and with width and length more than ten times larger.

The MFM experiments were carried out on an AttoDry 1000/SU Attocube scanning probe system, using an oscillating magnetic $\mathrm{Co} / \mathrm{Cr}$-coated cantilever (MESP, Bruker) excited at resonance by a dither. The artificial color in MFM maps encodes the phase shift in the cantilever oscillations, which is proportional to the magnetic field component perpendicular to the scanned surface. More detailed information can be found in Ref. [10].

\section{MICROWAVE CHARACTERIZATION TECHNIQUE}

The microwave characterization was performed by means of the CPWR technique, which consists in measuring the perturbations induced to the resonance of a CPWR by coupling a small sample to it [14] (for details, see Appendix A). In brief, measurements of the resonance curve were repeated with and without the crystal under study, and modifications of the resonance frequency $f_{0}$ and and quality factor $Q$ induced by the presence of the crystal (Fig. 1) were used to determine its complex magnetic susceptibility. It is composed of two parts [20]: the magnetic response of the bulk to an applied field $\left(\chi_{m}\right)$ and the response due to the screening effect. The latter is linked to the London penetration depth $\lambda_{L}$ and the quasiparticle microwave conductivity $\sigma_{n}$. For a superconducting slab of half-thickness $t$, with the rf magnetic field parallel to its broad face (i.e., parallel to $a b$ planes), the modifications in the

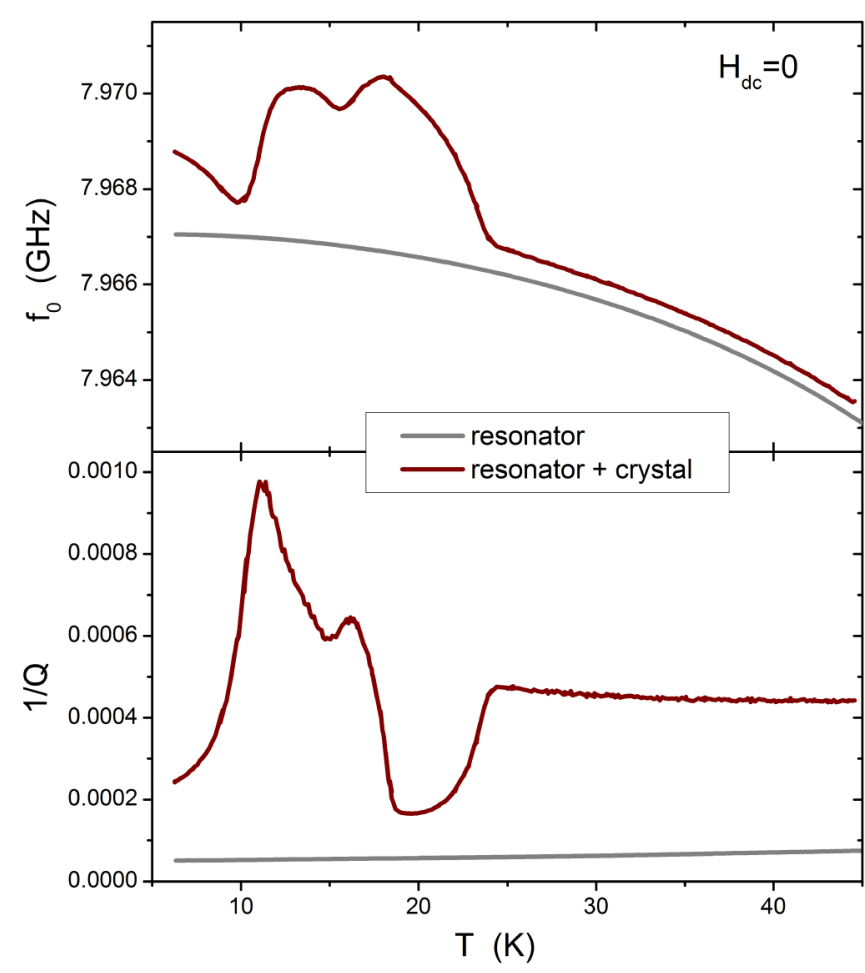

FIG. 1. Resonance frequency and inverse of the quality factor as a function of temperature for the empty resonator and for the resonator with the crystal coupled to it. 
resonance frequency $(\Delta f / f)$ and quality factor $(\Delta(1 / Q))$ can be written as (see Appendix $A$ for further details):

$$
\begin{aligned}
& 2 \frac{\Delta f}{f} \approx \Gamma_{f}\left\{1-\chi_{m}^{\prime}-\operatorname{Re}\left[\frac{\tanh (k t)}{k t}\right]\right\}, \\
& \Delta\left(\frac{1}{Q}\right) \approx \Gamma_{Q}\left\{\chi_{m}^{\prime \prime}+\operatorname{Im}\left[\frac{\tanh (k t)}{k t}\right]\right\},
\end{aligned}
$$

where $k=\sqrt{1 / \lambda_{L}^{2}+j \omega \mu_{0} \sigma_{n}}$. Thus, after a calibration, from experimental data and through Eqs. (1) and (2), $\lambda_{L}(T), \sigma_{n}(T)$, $\chi_{m}^{\prime}(T)$, and $\chi_{m}^{\prime \prime}(T)$ can be obtained. The dc magnetic field was applied parallel to the resonator plane and to the probe rf magnetic field, i.e., parallel to $a b$ planes of the crystals. Microwave susceptibility was measured either while warming up after the sample was cooled in zero field (zero-field cooling, ZFC) or during cooling in an applied field (field cooling, FC).

\section{RESULTS}

Figure 2 shows the results of the analysis done for $\mathrm{BaFe}_{2}\left(\mathrm{As}_{1-x} \mathrm{P}_{x}\right)_{2}$ and $\mathrm{EuFe}_{2}\left(\mathrm{As}_{1-x} \mathrm{P}_{x}\right)_{2}$ crystals. Figures 2(a) and 2(b) report the quantities $\left[1-2(\Delta f / f) / \Gamma_{f}\right]$ and $\Delta(1 / Q) / \Gamma_{Q}$, which represent respectively the real and the imaginary parts of the overall complex magnetic susceptibility, according to Eqs. (1) and (2) ( $\Gamma_{f}$ and $\Gamma_{Q}$ are geometrical

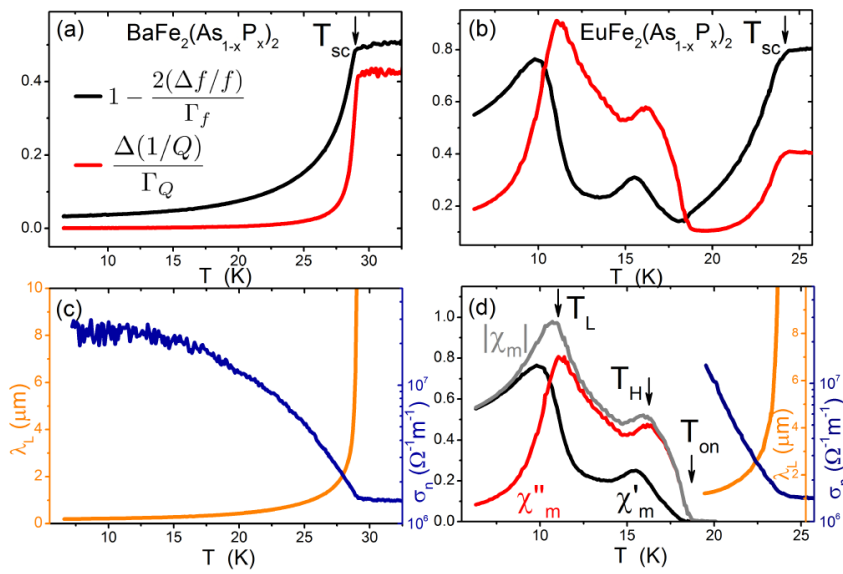

FIG. 2. Comparison between the microwave responses of $\mathrm{BaFe}_{2}\left(\mathrm{As}_{1-x} \mathrm{P}_{x}\right)_{2}[(\mathrm{a}),(\mathrm{c})]$ and $\mathrm{EuFe}_{2}\left(\mathrm{As}_{1-x} \mathrm{P}_{x}\right)_{2}[(\mathrm{~b}),(\mathrm{d})]$. In panels (a) and (b), black and red lines represent $\left[1-2(\Delta f / f) / \Gamma_{f}\right]$ and $\Delta(1 / Q) / \Gamma_{Q}$, respectively, where $\Delta f$ and $\Delta(1 / Q)$ are the modifications in the resonance frequency and quality factor of a CPWR, induced by the crystal under study $\left(\Gamma_{f}\right.$ and $\Gamma_{Q}$ are geometrical factors). The arrows indicate the superconductivity onset temperature, $T_{s c}$. Panel (c) shows the London penetration depth, $\lambda_{L}$, and the quasiparticle conductivity, $\sigma_{n}$, as a function of temperature, obtained by the procedure described in the text from the data reported in panel (a). The same is reported in panel (d) for the data shown in panel (b), between $T_{s c}$ and the onset of the magnetic signal, $T_{o n}$ (orange and blue curves and scales). At lower temperatures, a smooth baseline due to Meissner diamagnetism was subtracted from data in panel (b), and the real part $\chi_{m}^{\prime}$, the imaginary part $\chi_{m}^{\prime \prime}$, and the modulus $\left|\chi_{m}\right|$ of the bulk magnetic susceptibility were extracted according to the procedure described in the text [panel (d), black, red, and gray curves, left scale]. Other arrows indicate reference temperatures discussed in the text. factors used for calibration). The onset temperature of the superconducting transition, $T_{s c}$, is indicated by an arrow. In the case of the purely superconducting $\mathrm{BaFe}_{2}\left(\mathrm{As}_{1-x} \mathrm{P}_{x}\right)_{2}$ sample, it was then possible to extract the London penetration depth and the microwave quasiparticle conductivity, shown in Fig. 2(c), for all temperatures. In the case of $\mathrm{EuFe}_{2}\left(\mathrm{As}_{1-x} \mathrm{P}_{x}\right)_{2}$, the purely superconducting regime is visible only in the range between $T_{s c}$ and about $T=18 \mathrm{~K}$, hereafter labeled $T_{o n}$. Within this range, the same procedure leads to results for $\lambda_{L}$ and $\sigma_{n}$ qualitatively similar to those for the Ba122 sample, see the right part of Fig. 2(d), and quantitatively consistent with previous optical investigations [21,22]. Below $T_{o n}$, an additional signal due to a bulk susceptibility contribution emerges. The real and imaginary parts of the bulk complex susceptibility are shown in Fig. 2(d), as $\chi_{m}^{\prime}$ and $\chi_{m}^{\prime \prime}$ (left scale), together with the modulus $\left|\chi_{m}\right|$. Two distinct transitions, separated by about $6 \mathrm{~K}$, are visible in $\chi_{m}^{\prime}$, while accordingly, $\chi_{m}^{\prime \prime}$ shows two broad peaks. We discuss below the dependence on external parameters of three reference temperatures: the onset temperature of the bulk magnetic ordering, $T_{o n}$, and the temperatures of the two magnetic transitions, labeled as $T_{H}$ and $T_{L}$, all marked in Fig. 2(d) by arrows. It is evident that these reference temperatures can be deduced easily from rough data, $-\Delta f / f$ and $\Delta(1 / Q)$, therefore henceforth we only show plots with these quantities.

The effects of a dc field $H_{d c}$ applied parallel to $a b$ planes are reported in Fig. 3 for a $x=0.20$ crystal. Figure 3(a) shows $-\Delta f / f$ and $\Delta(1 / Q)$ as a function of temperature at $\mu_{0} H_{d c}=100 \mathrm{mT}$, both in ZFC and FC modes. The curves show hysteresis as soon as the bulk magnetic signal appears. In particular, the height of the $\Delta(1 / Q)$ peaks changes: The peak at lower temperature is higher than the peak at higher temperature for the ZFC mode, the opposite of the FC mode. Figure 3(b) shows the behavior of $\Delta(1 / Q)(T)$ for different values of $H_{d c}$. The peaks evolve differently: $T_{H}$ decreases while $T_{L}$ increases with field until they merge. Increasing the field even higher shifts the merged peak down, until it completely disappears from our observation window at about $500 \mathrm{mT}$. In Fig. 3(c), we report the $f$ and $1 / Q$ shifts as a function of $H_{d c}$ at $T=11 \mathrm{~K}$, for increasing and decreasing fields: Irreversibility is shown below $\mu_{0} H_{d c}=0.25 \mathrm{~T}$.

\section{DISCUSSION}

Generally, our data confirm the coexistence of superconductivity and magnetism in $\mathrm{EuFe}_{2}\left(\mathrm{As}_{1-x} \mathrm{P}_{x}\right)_{2}$ for temperatures below about $18 \mathrm{~K}$, as also shown by the dc magnetic characterization reported in Appendix B. The competition between superconducting and magnetic orders is evident from Fig. 4, where we report the $H_{d c}$ evolution of the three reference temperatures, $T_{o n}, T_{H}$, and $T_{L}$, for the crystal with doping $x=0.20$ and $T_{s c}=24.1 \mathrm{~K}$ [from data shown in Fig. 3(b)], and for the crystal with $x=0.23$ and $T_{s c}=22.3 \mathrm{~K}$. The crystal with weaker superconductivity (lower critical temperature) shows the magnetic transitions at higher temperatures and fields.

In order to get a microscopic insight into observed behavior, we performed MFM imaging on the $x=0.20$ crystal. The MFM maps acquired at different temperatures are presented in Fig. 5. Upon cooling, a striped quasi-one-dimensional 

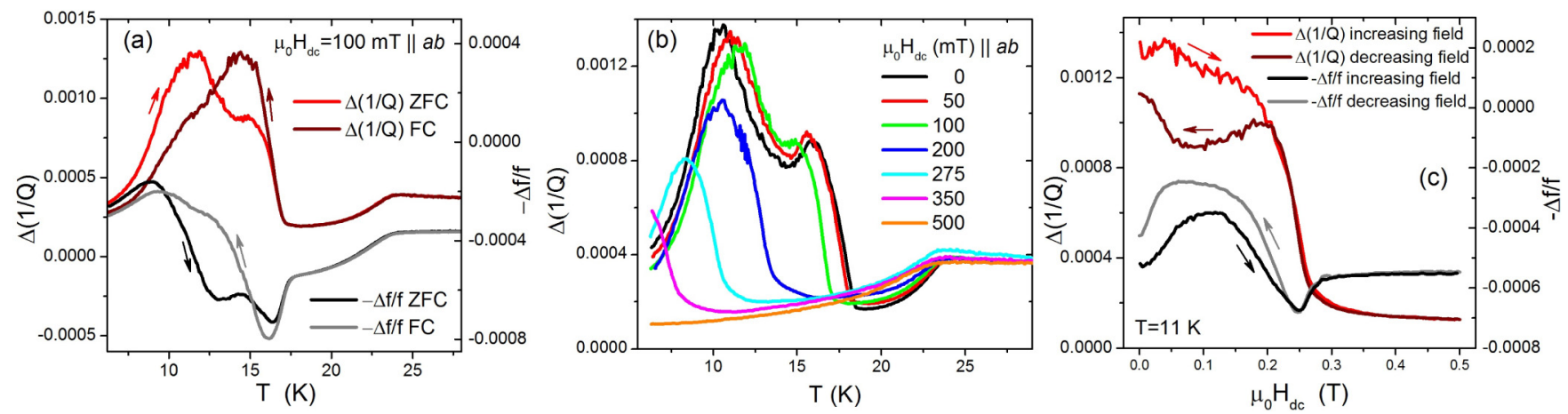

FIG. 3. Shifts of the quality factor and resonant frequency, $\Delta(1 / Q)$ and $-\Delta f / f$, as a function of temperature, for both ZFC and FC modes, in a dc field of $100 \mathrm{mT}$ applied parallel to $a b$ planes of the crystal with $x=0.20$ (a). FC-ZFC irreversibility emerges in the magnetic part of the response. Panel (b) shows $\Delta(1 / Q)$ as a function of temperature in ZFC mode for different values of the applied dc field, up to $500 \mathrm{mT}$. Shifts of the quality factor and resonant frequency are shown in panel (c) as a function of dc magnetic field at $T=11 \mathrm{~K}$, for increasing and decreasing fields.

(quasi-1D) ferromagnetic Meissner state [DMS, Fig. 5(a)] starts to transform to a domain vortex-antivortex (V-A) state (DVS) [10]. The latter is characterized by a more complex quasi-two-dimensional (quasi-2D) domain structure [Fig. 5(b)] that rapidly evolves with decreasing temperature [Figs. 5(c) and 5(d)]. The microwave $\chi_{m}^{\prime \prime}$ peak at $T_{H}$ coincides with this dimensionality transition. Below approximately $15 \mathrm{~K}$, the V-A domain structure evolves to a more regular striped V-A phase [Fig. 5(e)] which is characterized, at yet lower temperatures, by the appearance of finely zigzagging domain walls. Correspondingly, $\chi_{m}^{\prime}$ rises again. Below $12 \mathrm{~K}$, however, the pattern evolution "freezes" and does not change anymore down to $4.35 \mathrm{~K}$ (and rf susceptibility drops down). The thermal freezing-defreezing is clearly seen upon thermal cycling [compare Figs. 5(d) with 5(g) and Figs. 5(e) with

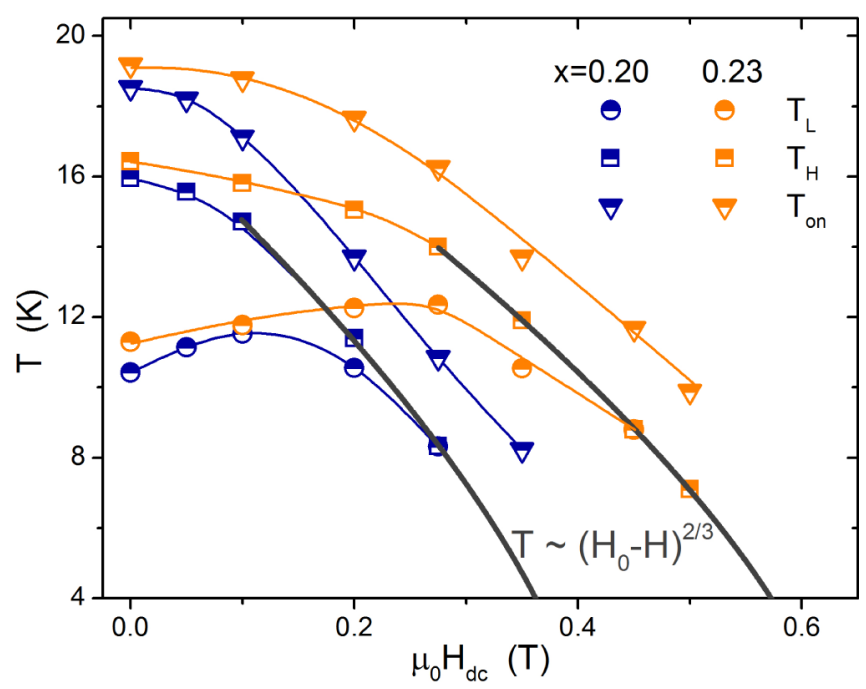

FIG. 4. Evolution with magnetic field of the three reference temperatures, $T_{o n}, T_{H}$, and $T_{L}$, for two $\mathrm{EuFe}_{2}\left(\mathrm{As}_{1-x} \mathrm{P}_{x}\right)_{2}$ crystals with $x=0.20$ and 0.23 and $T_{s c}=24.1 \mathrm{~K}$ and $22.3 \mathrm{~K}$, respectively. Blue and orange lines are guides to the eye. The dark gray lines display the $T \sim\left(H_{0}-H\right)^{2 / 3}$ dependence expected for a QCP, with $\mu_{0} H_{0}=0.41 \mathrm{~T}$ and $0.63 \mathrm{~T}$ for $x=0.20$ and 0.23 , respectively. 5(h)]. This thermal hysteresis shown in MFM images is not clearly revealed in rf susceptibility with $H_{d c}=0$, probably due to rf-stimulated domain and vortex shaking [23-25].

Thus, the peak shown by rf- $\chi_{m}^{\prime \prime}$ at $T_{L}$ is peculiar of the high-frequency regime, not clearly revealed by MFM and dc analysis (Appendix B). It could be attributed to rf-induced
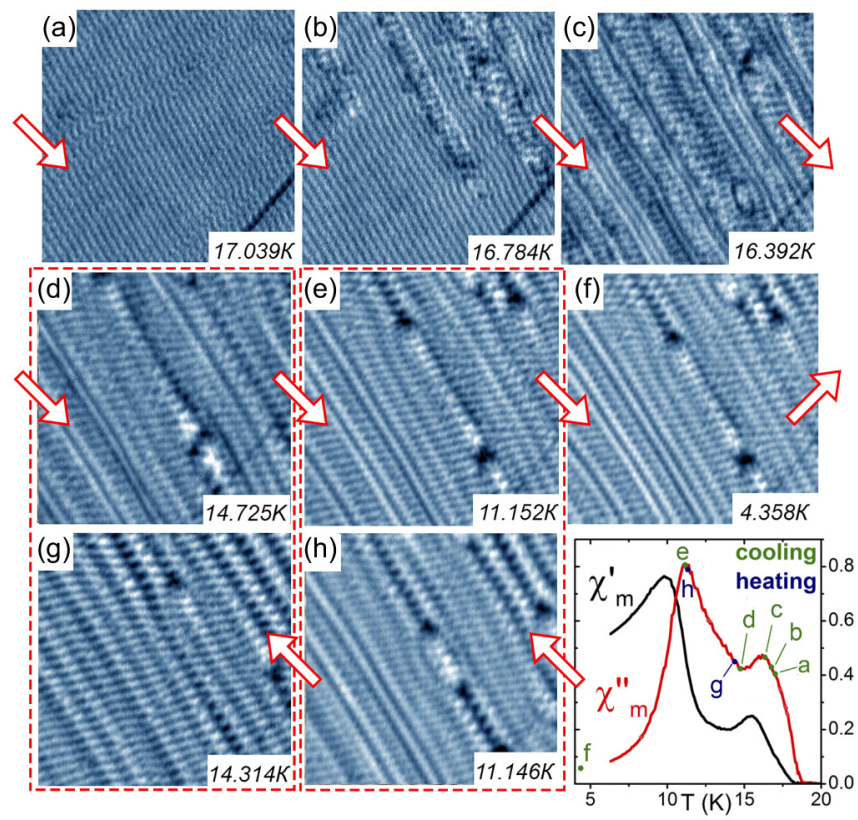

FIG. 5. MFM maps acquired on a $x=0.20$ crystal at temperatures below $T_{s c}$ and their link to peaks observed in microwave data. The maps reveal spatial variations of the out-of-plane component of the local magnetic field. Maps [(a)-(f)] correspond to the sample cooling [(f), (h), (g)] to heating. (a) Below Curie temperature, linear ferromagnetic Meissner domains form a short-period quasi-1D pattern. [(b)-(d)] Transition from DMS to DVS, between $16.8 \mathrm{~K}$ and $15 \mathrm{~K}$, is characterized by the proliferation of DVS domains of a larger period and a more complex 2D organization. [(d), (g), (e), (h)] A hysteretic behavior is observed in maps acquired around $8-15 \mathrm{~K}$ upon temperature cycling (red dashed lines delimit pairs of maps acquired at close temperatures). (f) Below $8 \mathrm{~K}$ the DVS pattern is "frozen." 
dynamics, related to vortex-antivortex motion in corresponding domains, as well as to FM domain motion. It could also be related to a spin reorientation transition of the $\mathrm{Eu}^{2+}$ canted ferromagnetic subsystem (spin canting does not give contrast in MFM maps). A spin glass transition should probably be excluded for $\operatorname{EuFe}_{2}\left(\mathrm{As}_{1-x} \mathrm{P}_{x}\right)_{2}$, at least in the investigated doping range, since we observed by MFM a well-defined domain structure, i.e., evidence of long-range ferromagnetic ordering [10].

Note that the origin of the thermal irreversibility observed in MFM images (Fig. 5) and in in-field rf susceptibility [Fig. 3(a)] cannot be decided at this stage, since it can be attributed to several causes [26], e.g., domain wall pinning [27], geometrical barriers [12], or in general to the FM-AFM competition at the macroscopic level, that need to be checked in further works.

The evolution of the $\chi_{m}^{\prime \prime}$ peaks with in-plane magnetic field could suggest the presence of a quantum critical point (QCP), i.e., of a $T=0$ quantum phase transition driven by a nonthermal control parameter. In order to check whether our data are compatible with the presence of a QCP, we fitted them with the same scaling law $T \sim\left(H_{0}-H\right)^{2 / 3}$ reported in Ref. [28] for heavy-fermion antiferromagnets and interpreted as an evidence of SDW-type QCP. In fact, in these systems the field dependence of the Néel temperature for $H \| a$ shows a first increase [28-30], as we report for $T_{L}$ in Fig. 4 . The fit gives $\mu_{0} H_{0}=0.4-0.6 \mathrm{~T}$ for our crystals, depending on doping $x$ (Fig. 4). These values interestingly correspond to $T=0$ transitions reported in literature: In Ref. [31], $0.6 \mathrm{~T}$ is the in-plane magnetic field that at $T \rightarrow 0$ causes the transition from $c$-axis-canted to $a b$-aligned magnetic moments in the $\mathrm{Eu}^{2+}$ subsystem. On the other hand, a metamagnetic-like change at a field $\mu_{0} H_{a p p} \| a \approx 0.5-0.7 \mathrm{~T}$ was observed in other $\mathrm{Eu}^{2+}$ systems (EuGa4) for $T \rightarrow 0$, as antiferromagnetic domains turned into a single domain [32]. Therefore, although the fit only gives an indication since the number of data points is too low to draw rigorous conclusions, our data seem to be compatible with the presence of a QCP. The nature of this possible QCP, whether due to spin canting or domain rearrangement, remains undecided and deserves further investigation.

\section{CONCLUSIONS}

In summary, we showed that the analysis of the complex susceptibility at microwave frequencies of $\mathrm{EuFe}_{2}\left(\mathrm{As}_{1-x} \mathrm{P}_{x}\right)_{2}$ single crystals reveals peculiar features. In particular, below the superconducting critical temperature two magnetic transitions are indicated by peaks of the imaginary part of susceptibility, $\chi_{m}^{\prime \prime}$. The comparison with MFM maps allowed us to associate the $\chi_{m}^{\prime \prime}$ peak at about $17 \mathrm{~K}$ to a dimensionality transition from quasi-unidimensional striped domains to more complex two-dimensional vortex-antivortex domain structure. Moreover, the bulky character of this transition, only suggested in Ref. [10], is now confirmed by the microwave bulk-sensitive analysis. The low-temperature $\chi_{m}^{\prime \prime}$ peak, specific to the high-frequency characterization, corresponds to a transition between different vortex-antivortex dynamical regimes, to a spin reorientation transition of the $\mathrm{Eu}^{2+}$ canted ferromagnetic subsystem, or to an interplay between the two phenomena.

The investigation of the field dependence of these magnetic transitions suggests the presence of a QCP (as in other 122 and 1144 systems [33-35]) in a field range of 0.4-0.6 T. We believe that the present work will stimulate further experiments and theories toward a comprehensive understanding of the behavior of domains and spin orientation in systems with coexisting superconductivity and ferromagnetism.

\section{ACKNOWLEDGMENTS}

This work was partially supported by the Italian Ministry of Education, University and Research (Project PRIN "HIBiSCUS," Grant No. 201785KWLE), by the National Science Foundation of China (No. 11474252, No. 1611140101, and No. U1432135), and by a Grant-in-Aid for Scientific Research (A) (Grant No. 17H01141) from the Japan Society for the Promotion of Science (JSPS). MFM studies were supported by the Russian Scientific Foundation (RSF) (Grant No. 18-72-10118)

\section{APPENDIX A: ADDITIONAL INFORMATION ON THE MICROWAVE TECHNIQUE}

\section{Experimental details}

The CPWR used for our microwave measurements consists of a patterned $\mathrm{YBa}_{2} \mathrm{Cu}_{3} \mathrm{O}_{7-x}$ film with a thickness of 250 $\mathrm{nm}$, grown on an $\mathrm{MgO}$ substrate. The central conductor strip has a width of $350 \mu \mathrm{m}$ and the distance between the ground planes is $650 \mu \mathrm{m}$. The critical temperature of the YBCO film is about $88 \mathrm{~K}$, and its zero-temperature penetration depth is $155 \mathrm{~nm}$ [36]. Resonance frequency $f_{0}$ and unloaded quality factor $Q$ were evaluated from measurements of the resonance curves (examples in Fig. 6) obtained in a Cryomech PT 415 pulse tube cooler by means of a Rohde Schwarz ZVK vector network analyzer for an input power of $-10 \mathrm{dBm}$, below the nonlinearity threshold for the resonator. The dc magnetic field was applied parallel to the resonator plane by means of an external cryomagnetic cryogen free superconducting magnet. The crystal under study was coupled to the resonator by fixing

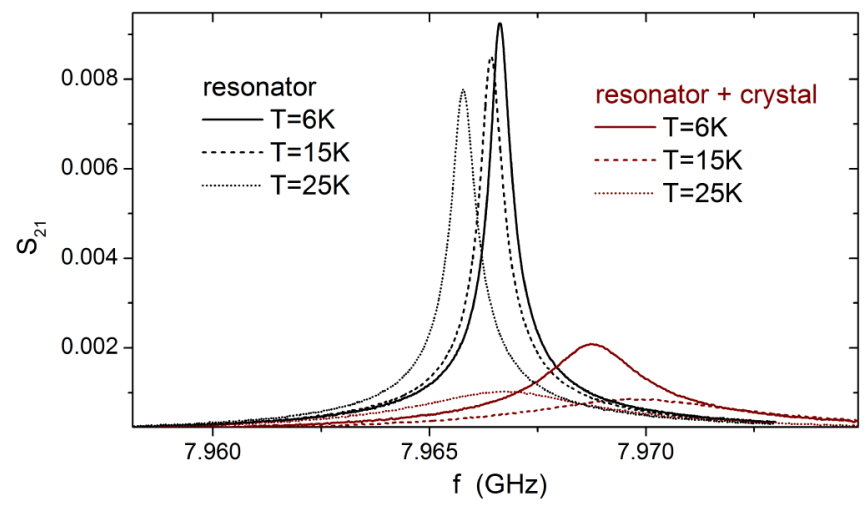

FIG. 6. Examples of resonance curves for the empty resonator and for the resonator with the crystal coupled to it. 


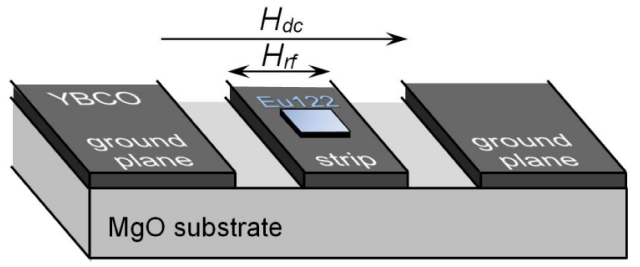

FIG. 7. Experimental configuration (not to scale).

it on top of the central strip line by means of a small amount of high-vacuum grease (see the sketch in Fig. 7). The crystal was positioned in the center of the strip far from edges, in a region where rf magnetic fields are quite uniform and rf electric fields are negligible [14].

\section{Data analysis}

A resonator is characterized by its complex angular frequency $\omega=\omega^{\prime}+j \omega^{\prime \prime}$, and it is possible to express the quality factor of the resonance and the resonance frequency, respectively, as [37] $Q=\omega^{\prime} /\left(2 \omega^{\prime \prime}\right)$ and $f=\omega^{\prime} /(2 \pi)$. When a sample is coupled to the resonator, the complex permittivity and/or complex permeability of the material can be determined from the changes of $Q$ and $f$. Provided the sample is much smaller than the effective cavity and the electromagnetic field outside the sample does not change significantly, the angular frequency shift due to the sample can be calculated as [37]

$$
\frac{\omega-\omega_{0}}{\omega}=-\frac{\int_{V_{s}}\left(\Delta \varepsilon \boldsymbol{E} \cdot \boldsymbol{E}_{0}^{*}+\Delta \mu \boldsymbol{H} \cdot \boldsymbol{H}_{0}^{*}\right) d V}{\int_{V_{c}}\left(\varepsilon_{0} \boldsymbol{E} \cdot \boldsymbol{E}_{0}^{*}+\mu_{0} \boldsymbol{H} \cdot \boldsymbol{H}_{0}^{*}\right) d V},
$$

where the index 0 indicates the absence of the sample coupled to the resonator. The asterisks denote the complex conjugates, and $V_{s}$ and $V_{c}$ are the sample and cavity volumes, respectively. If the sample is positioned in a region where $\boldsymbol{E} \approx 0$, and $\boldsymbol{H} \approx$ $\boldsymbol{H}_{0}$, and assuming that $\omega^{\prime} \approx \omega_{0}^{\prime}$ and $Q, Q_{0} \gg 1$, the equation

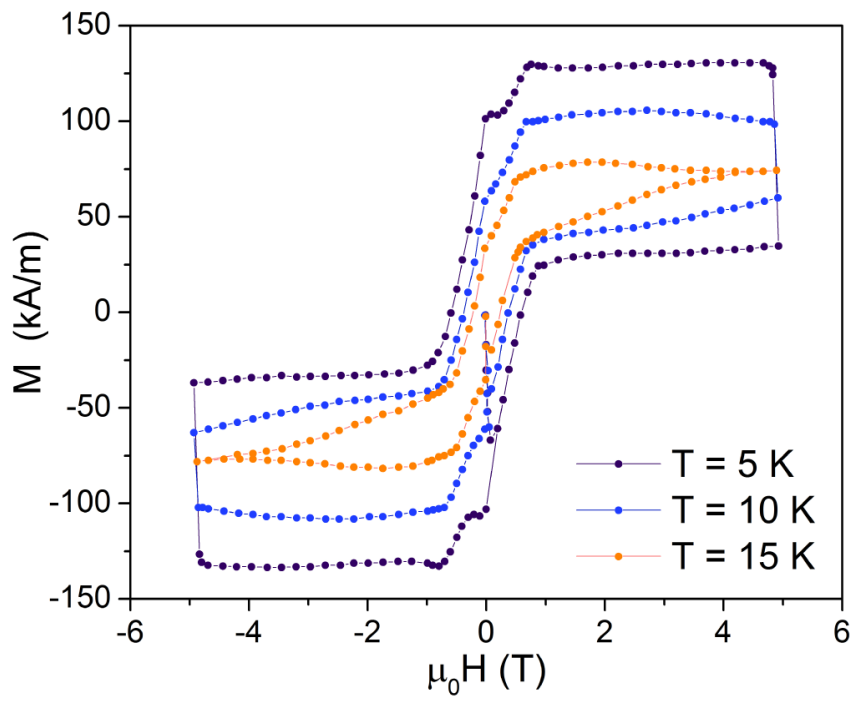

FIG. 8. $M(H)$ hysteresis loops for a $\mathrm{EuFe}_{2}\left(\mathrm{As}_{1-x} \mathrm{P}_{x}\right)_{2}$ crystal with $x=0.20$. above gives

$$
\begin{aligned}
& \left(\frac{f-f_{0}}{f}\right)+j\left(\frac{1}{2 Q}-\frac{1}{2 Q_{0}}\right) \\
& \approx-\frac{\int_{V_{s}}\left(\mu-\mu_{0}\right)\left|\boldsymbol{H}_{0}\right|^{2} d V}{2 \int_{V_{c}} \mu_{0}\left|\boldsymbol{H}_{0}\right|^{2} d V} \approx-\frac{1}{2} \Gamma\left(\chi_{m}+\chi_{s}\right),
\end{aligned}
$$

where $\Gamma \approx V_{s} / V_{c}$ resumes the geometrical factors connected to the distribution of the fields in the cavity. As mentioned above, the complex magnetic susceptibility is composed of two parts: the magnetic response of the bulk to an applied field $\left(\chi_{m}\right)$ and the response due to the screening effect $\left(\chi_{s}\right)$. For a superconducting slab of half-thickness $t$, with the rf magnetic field parallel to its broad face (i.e., parallel to $a b$ planes), at low temperatures and with negligible losses, $\chi_{s}^{\prime} \approx$ $-\left(1-\lambda_{L} / t\right)$ and $\chi_{s}^{\prime \prime} \approx 0$, where $\lambda_{L}$ is the London penetration depth. If losses are not negligible and the full temperature range is considered, Eq. (A1) can be rewritten, for its real and imaginary parts, as reported above in the main text [Eqs. (1) and (2)]. Two different geometrical factors are considered in these equations, $\Gamma_{f}$ and $\Gamma_{Q}$, since they are expected to be slightly different from each other, due to the adopted approximations [37]. The geometrical factors cannot be calcu-
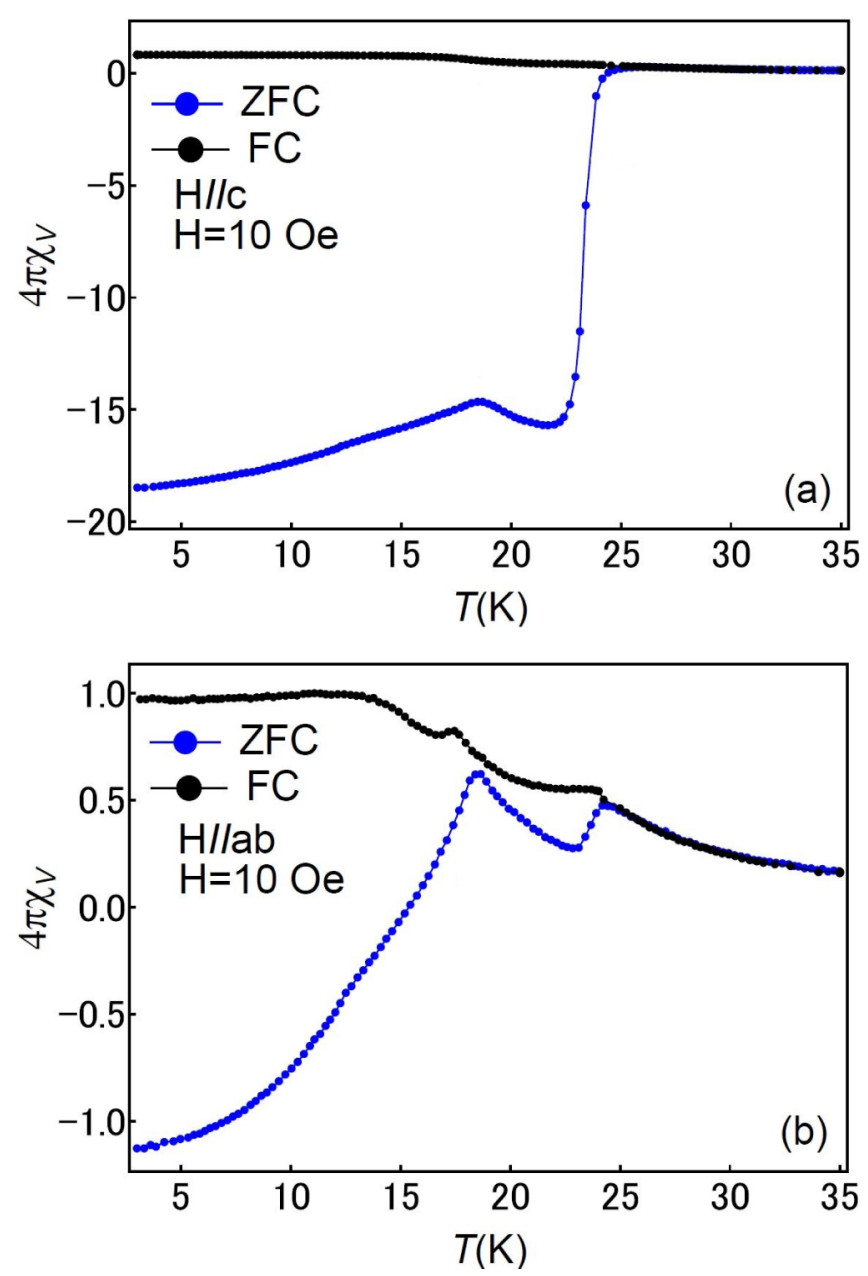

FIG. 9. Temperature dependence of external volume susceptibility in a 10 Oe field applied parallel to $c$ axis (a) and to $a b$ planes (b), measured by a SQUID magnetometer. 
lated with the needed precision, especially for the planar geometry of our resonator; rather, they should be determined in a self-consistent way from data above $T_{s c}$, where the crystals show a metallic behavior without bulk magnetic response. In fact, for a metal $\operatorname{Re}(k)=\operatorname{Im}(k)=1 / \delta$, where $\delta=\sqrt{2 / \omega \mu \sigma}$ is the classical skin depth. This, in combination with Eqs. (1) and (2), allows one to determine $\Gamma_{f}$ and $\Gamma_{Q}$ by fitting $\Delta f / f$ and $\Delta(1 / Q)$ data, with the constraint to keep the same $\delta(T)$ in both the cases. In Ref. [14], we discussed in detail the calibration procedure and how to take into account the finite size of the crystal in the equations above, with the consequent demagnetization effects. Then, from Eqs. (1) and (2), $\lambda_{L}(T)$, $\sigma_{n}(T), \chi_{m}^{\prime}(T)$, and $\chi_{m}^{\prime \prime}(T)$ can be obtained.

\section{APPENDIX B: DC MAGNETIC CHARACTERIZATION}

Magnetization versus magnetic field $M(H)$ curves acquired at various temperatures with the magnetic field directed parallel to the $c$ axis of the crystal are shown in
Fig. 8. The shape of the loops can be understood in terms of a superposition of typical hysteresis loops of a type-II superconductor and the magnetization of $\mathrm{Eu}^{2+}$ ferromagnetic subsystem.

Figure 9 shows the temperature dependence of susceptibility in a 10 Oe field applied parallel to $c$ axis [Fig. 9(a)] and to $a b$ planes [Fig. 9(b)] for a $\operatorname{EuFe}_{2}\left(\mathrm{As}_{1-x} \mathrm{P}_{x}\right)_{2}$ crystal with $x=0.20$, measured by a SQUID magnetometer [38]. An evident feature is shown at about $18 \mathrm{~K}$, where the susceptibility first increases upon cooling and then shows a peak, unlike in nonmagnetic superconductors. The onset of magnetic ordering and the peak correspond to those obtained by the microwave characterization reported in the paper $\left(T_{o n}\right.$ and $T_{H}$ ). Conversely, no clear correspondence is shown with the low-temperature peak of the imaginary part of the bulk microwave susceptibility. In fact, only a very faint feature could be seen in Fig. 9(b) at about $12 \mathrm{~K}$, as a weak change of slope in the ZFC curve, but the microwave peak is shown at a slightly lower temperature.
[1] I. I. Mazin, D. J. Singh, M. D. Johannes, and M. H. Du, Phys. Rev. Lett. 101, 057003 (2008).

[2] G. Ghigo, D. Torsello, G. A. Ummarino, L. Gozzelino, M. A. Tanatar, R. Prozorov, and P. C. Canfield, Phys. Rev. Lett. 121, 107001 (2018).

[3] G. Ghigo, G. A. Ummarino, L. Gozzelino, R. Gerbaldo, F. Laviano, D. Torsello, and T. Tamegai, Sci. Rep. 7, 13029 (2017).

[4] D. Torsello, G. A. Ummarino, L. Gozzelino, T. Tamegai, and G. Ghigo, Phys. Rev. B 99, 134518 (2019).

[5] S. Zapf, H. S. Jeevan, T. Ivek, F. Pfister, F. Klingert, S. Jiang, D. Wu, P. Gegenwart, R. K. Kremer, and M. Dressel, Phys. Rev. Lett. 110, 237002 (2013).

[6] S. Zapf and M. Dressel, Rep. Prog. Phys. 80, 016501 (2016).

[7] Y. Xiao, Y. Su, M. Meven, R. Mittal, C. M. N. Kumar, T. Chatterji, S. Price, J. Persson, N. Kumar, S. K. Dhar, A. Thamizhavel, and T. Brueckel, Phys. Rev. B 80, 174424 (2009).

[8] Z. Ren, Q. Tao, S. Jiang, C. Feng, C. Wang, J. Dai, G. Cao, and Z. Xu, Phys. Rev. Lett. 102, 137002 (2009).

[9] W. T. Jin, M. Meven, A. P. Sazonov, S. Demirdis, Y. Su, Y. Xiao, Z. Bukowski, S. Nandi, and T. Brückel, Phys. Rev. B 99, 140402(R), (2019).

[10] V. S. Stolyarov, I. S. Veshchunov, S. Y. Grebenchuk, D. S. Baranov, I. A. Golovchanskiy, A. G. Shishkin, N. Zhou, Z. Shi, X. Xu, S. Pyon, Y. Sun, W. Jiao, G.-H. Cao, L. Y. Vinnikov, A. A. Golubov, T. Tamegai, A. I. Buzdin, and D. Roditchev, Sci. Adv. 4, eaat1061 (2018).

[11] L. Y. Vinnikov, I. S. Veshchunov, M. S. Sidel'nikov, V. S. Stolyarov, S. V. Egorov, O. V. Skryabina, W. Jiaod, G. Cao, and T. Tamegai, JETP Lett. 109, 521 (2019).

[12] Z. Devizorova, S. Mironov, and A. Buzdin, Phys. Rev. Lett. 122, 117002 (2019).

[13] A. Pogrebna, T. Mertelj, N. Vujičić, G. Cao, Z. Xu, and D. Mihailovic, Sci. Rep. 5, 7754 (2015).

[14] G. Ghigo, G. A. Ummarino, L. Gozzelino, and T. Tamegai, Phys. Rev. B 96, 014501 (2017).
[15] G. Ghigo, R. Gerbaldo, L. Gozzelino, F. Laviano, and T. Tamegai, IEEE Trans. Appl. Supercond. 26, 7300104 (2016).

[16] G. Ghigo, D. Torsello, R. Gerbaldo, L. Gozzelino, F. Laviano, and T. Tamegai, Supercond. Sci. Technol. 31, 034006 (2018).

[17] D. Torsello, K. Cho, K. R. Joshi, S. Ghimire, G. A. Ummarino, N. M. Nusran, M. A. Tanatar, W. R. Meier, M. Xu, S. L. Bud'ko, P. C. Canfield, G. Ghigo, and R. Prozorov, Phys. Rev. B 100, 094513 (2019).

[18] X. Xu, W. H. Jiao, N. Zhou, Y. K. Li, B. Chen, C. Cao, J. Dai, A. F. Bangura, and G. Cao, Phys. Rev. B 89, 104517 (2014).

[19] A. Park, S. Pyon, Y. Sun, I. Veshchunov, J. Chen, N. Ito, T. Suwa, T. Tamegai, H. Kitamura, and A. Ichinose, Phys. Rev. B 98, 054512 (2018).

[20] M. D. Vannette, A. S. Sefat, S. Jia, S. A. Law, G. Lapertot, S. L. Bud'ko, P. C. Canfield, J. Schmalian, and R. Prozorov, J. Magnetism Magn. Mat. 320, 354 (2008).

[21] D. Neubauer, A. V. Pronin, S. Zapf, J. Merz, H. S. Jeevan, W.-H Jiao, P. Gegenwart, G.-H. Cao, and M. Dressel, Phys. Status Solidi B 254, 1600148 (2017).

[22] D. Wu, G. Chanda, H. S. Jeevan, P. Gegenwart, and M. Dressel, Phys. Rev. B 83, 100503(R) (2011).

[23] E. H. Brandt and G. P. Mikitik, Phys. Rev. Lett. 89, 027002 (2002).

[24] G. Ghigo, F. Laviano, L. Gozzelino, R. Gerbaldo, E. Mezzetti, E. Monticone, and C. Portesi, J. Appl. Phys. 102, 113901 (2007).

[25] I. A. Golovchanskiy, N. N. Abramov, V. S. Stolyarov, V. V. Bolginov, V. V. Ryazanov, A. A. Golubov, and A. V. Ustinov, Adv. Funct. Mater. 28, 1802375 (2018).

[26] B. Maji, K. Suresh, and A. Nigam, J. Phys.: Condens. Matter 23, 506002 (2011)

[27] B. Chevalier, R. Poettgen, B. Darriet, P. Gravereau, and J. Etourneau, J. Alloys Comp. 233, 150 (1996).

[28] L. Jiao, Y. Chen, Y. Kohama, D. Graf, E. Bauer, J. Singleton, J.-X. Zhu, Z. Weng, G. Pang, T. Shang et al., Proc. Natl. Acad. Sci. U.S.A. 112, 673 (2015). 
[29] A. L. Cornelius, P. G. Pagliuso, M. F. Hundley, and J. L. Sarrao, Phys. Rev. B 64, 144411 (2001).

[30] Y. Krupko, A. Demuer, S. Ota, Y. Hirose, R. Settai, and I. Sheikin, Phys. Rev. B 93, 085121 (2016).

[31] S. Nandi, W. T. Jin, Y. Xiao, Y. Su, S. Price, D. K. Shukla, J. Strempfer, H. S. Jeevan, P. Gegenwart, and T. Brückel, Phys. Rev. B 89, 014512 (2014).

[32] A. Nakamura, Y. Hiranaka, M. Hedo, T. Nakama, Y. Miura, H. Tsutsumi, A. Mori, K. Ishida, K. Mitamura, Y. Hirose et al., J. Phys. Soc. Jpn. 82, 104703 (2013).

[33] W. Zhang, Y. Wei, T. Xie, Z. Liu, D. Gong, X. Ma, D. Hu, P. Čermák, A. Schneidewind, G. Tucker et al., Phys. Rev. Lett. 122, 037001 (2019).
[34] H. Kim, M. A. Tanatar, C. Martin, E. C. Blomberg, N. Ni, S. L. Bud'ko, P. C. Canfield, and R. Prozorov, J. Phys.: Condensed Matter 30, 225602 (2018).

[35] Q.-P. Ding, W. R. Meier, J. Cui, M. Xu, A. E. Böhmer, S. L. Bud'ko, P. C. Canfield, and Y. Furukawa, Phys. Rev. Lett. 121, 137204 (2018).

[36] G. Ghigo, F. Laviano, R. Gerbaldo, and L. Gozzelino, Supercond. Sci. Technol. 25, 115007 (2012).

[37] L.-F. Chen, C. Ong, C. Neo, V. Varadan, and V. K. Varadan, Microwave Electronics: Measurement and Materials Characterization (John Wiley \& Sons, New York, 2004).

[38] I. Veshchunov, L. Y. Vinnikov, V. Stolyarov, N. Zhou, Z. Shi, X. Xu, S. Y. Grebenchuk, D. S. Baranov, I. Golovchanskiy, S. Pyon et al., JETP Lett. 105, 98 (2017). 